\title{
Développement durable. Dynamiques des territoires ruraux et logiques d'acteurs
}

\section{Abdelillah Hamdouch}

\section{(2) OpenEdition}

1 Journals

\section{Édition électronique}

URL : http://journals.openedition.org/economierurale/2855

DOI : 10.4000/economierurale.2855

ISSN : 2105-2581

\section{Éditeur}

Société Française d'Économie Rurale (SFER)

\section{Édition imprimée}

Date de publication : 1 novembre 2010

Pagination : 4-8

ISSN : 0013-0559

\section{Référence électronique}

Abdelillah Hamdouch, « Développement durable. Dynamiques des territoires ruraux et logiques d'acteurs », Économie rurale [En ligne], 320 | novembre-décembre 2010, mis en ligne le 01 novembre 2010, consulté le 01 mai 2019. URL : http://journals.openedition.org/economierurale/2855 ; DOI : 10.4000/economierurale.2855 


\title{
Développement durable Dynamiques des territoires ruraux et logiques d'acteurs
}

\author{
Abdelillah HAMDOUCH • Université de Lille 1, Centre lillois d'études et de recherches \\ sociologiques et économiques, CLERSÉ-MESHS-CNRS, et Centre de recherche sur l'entreprise, \\ CEREN, ESC Dijon - Bourgogne \\ Abdel.Hamdouch@univ-lille1.fr
}

$\mathbf{L}^{\mathrm{s}}$ es problématiques liées au développement durable (DD) recouvrent des spécificités et des enjeux différenciés selon la nature, l'échelle et les caractéristiques structurelles des territoires concernés. Territoires urbains versus territoires ruraux ou « rurbains », échelle nationale versus échelle régionale ou locale, territoires fragiles ou menacés versus territoires permissifs ou avantagés sont ainsi devenus des points d'entrée structurant les analyses théorique et empirique des dynamiques territoriales de DD. Parallèlement, la recherche d'équilibres ou de compromis praticables entre les trois piliers du DD (économique, social et environnemental) dans la mise en œuvre de politiques de développement territorial durable fait apparaître des configurations institutionnelles, culturelles et politiques extrêmement variées en termes de jeux d'acteurs et de rapports de pouvoir. Ces configurations résultent à la fois de l'histoire longue des territoires concernés (effet de « dépendance au sentier ») et des actions volontaires ou intentionnelles entreprises (ou susceptibles d'être engagées) afin d'introduire des changements, allant de simples inflexions à des bifurcations plus radicales, dans les trajectoires de développement suivies ou envisageables. Pris ensemble, ces facteurs de spécificité des territoires et de leurs dynamiques d'évolution déterminent ainsi très largement les possibilités et les changements effectifs en faveur d'un développement territorial plus durable.

Ce constat, a priori trivial, mérite cependant qu' on s'y arrête de manière attentive tant les conditions des changements structurels requis sont généralement complexes et porteuses d'incertitudes, voire de contradictions. Ainsi, que ce soit au niveau des mentalités, des comportements, des politiques et des pratiques, les logiques individuelles et collectives d'action ou, au contraire, de résistances favorables (respectivement opposées) à des modes de développement territorial plus durables font apparaître de redoutables problèmes de conflits d'intérêts, de rationalités, de visions qui conditionnent fondamentalement le sens, l'ampleur et la légitimité des changements institutionnels requis en faveur du DD (Hamdouch, 2005 ; Hamdouch et Zuindeau, 2010) ${ }^{1}$.

Si ces problèmes se posent inévitablement quelles que soient la nature et l'échelle du territoire considéré (Hamdouch et al., 2010) ${ }^{2}$, ils revêtent des formes de manifestation et des dynamiques de résolution spécifiques en fonction des caractéristiques économiques (niveau de développement, nature et diversité des activités, pouvoir d'achat, etc.), sociales (niveau

1. Hamdouch A. (2005). Émergence et légitimité des institutions, coordination économique et nature de la rationalité des agents. In "Innovation: the European Journal of Social Science Research", vol. $18, n^{\circ} 2$, p. 227-259. Hamdouch A., Zuindeau B. (2010). Sustainable Development, 20 years on: methodological innovations, practices, and open issues. In Hamdouch A. and Zuindeau B. (Eds.), "New Perspectives on Sustainable Development", Journal of Environmental Planning and Management, Special Issue, vol. 53, $\mathrm{n}^{\circ} 4$.

2. Hamdouch A., Parra C., Zuindeau B. (Éds.) (2010). Éclairages récents en économie de l'environnement et du développement durable : diversité territoriale et dynamiques socio-institutionnelles. Numéro spécial de Géographie, Économie, Société, à paraître. 
d'éducation, mode de vie, degré de cohésion, etc.) et environnementales (milieu naturel, diversité et qualité des ressources, opportunités de valorisation, etc.) de chaque type de territoire.

C'est précisément à travers ce prisme que se positionne le présent dossier d'Économie rurale qui se focalise sur l'analyse spécifique des dynamiques et politiques de développement durable dans des contextes territoriaux à la fois ruraux et soumis à des problèmes importants de développement. Dans le contexte français (métropolitain), les caractéristiques et les problèmes distinctifs des territoires ruraux sont aujourd'hui bien identifiés (Datar 2003 ; Sénat, 2008) ${ }^{3}$. De même, la problématique de la durabilité dans le contexte des pays en développement fait apparaître des approches et des dynamiques à la fois particulières et très variées (Boidin, Djeflat ; 2009) $)^{4}$.

Ces deux dimensions ont été très largement abordées lors du colloque international «La problématique du développement durable vingt ans après : nouvelles lectures théoriques, innovations méthodologiques et domaines d'extension » ${ }^{5}$, et dont sont issues les contributions réunies dans ce numéro. En effet, les territoires ruraux en général, et dans les pays en développement de manière plus frappante encore, présentent des caractéristiques propres qui en compliquent singulièrement la gestion durable :

3. Datar. (2003). Quelle France rurale pour 2020 ? Contribution à une nouvelle politique de développement rural durable. Paris, La Documentation française, 64 p. Sénat Rapport d'information fait au nom de la Délégation à l'aménagement et au développement durable du territoire sur le nouvel espace rural français (2008), $\mathrm{n}^{\circ} 468,15$ juillet, $151 \mathrm{p}$. 4. Boidin B., Djeflat A. (Éds.) (2009). Le développement durable : quelle appropriation dans les pays en développement ? Numéro spécial, Monde en développement, $\mathrm{n}^{\circ} 148$.

5. Lille, 20-22 novembre 2008. Présentation et programme : http://clerse.univ-lille1.fr/site_clerse/pages/ ActualitesEtColloques/DevDurable/pdf/programme_ detaille.pdf une grande diversité des environnements naturels (forêts, savanes, montagnes, brousses, etc.) ; des usages plus ou moins rivaux (et souvent conflictuels) des espacesressources (cultures, élevages, exploitations forestière... versus zones de biodiversité et espaces protégés) ; des problèmes de niveau de développement et la manifestation de contradictions souvent importantes entre les trois piliers du développement durable ; le poids des diversités ethniques et des coutumes ; la nature des acteurs en présence et la place accordée aux populations locales dans les négociations et la gouvernance des projets de développement du territoire; etc.

Ces différentes dimensions constituent la toile de fond des contributions composant ce dossier :

- L'article de Jean-Michel Sourisseau, Gilles Pestaña, Vincent Géronimi et Patrick Schembri s'intéresse à la manière dont s'insère la problématique du DD dans les politiques publiques et leur impact dans le contexte néo-calédonien.

- Pour leur part, Pierre-Marie Bosc, MarieHélène Dabat et Élodie Maître d'Hôtel procèdent, dans leur étude, à une analyse comparative des dynamiques de mise en œuvre du DD au Mali et à Madagascar.

- Enfin, Christophe Rouxel consacre son article à une étude précise des liens entre conservation de la biodiversité et développement durable des territoires, en s'appuyant sur le cas très parlant du Parc du W en Afrique de l'Ouest (à cheval entre le Bénin, le Burkina-Faso et le Niger) et des stratégies agraires et paysagères susceptibles de prendre place dans sa zone tampon.

Les deux premiers articles s'appuient très largement sur la participation des auteurs au projet Propocid (2007-2009) ${ }^{6}$

6. Propocid : La production des politiques de développement durable dans leurs contextes : construction de compromis institutionnels et ajustements temporels entre le global et le local, projet du Programme fédérateur de l'ANR « Agriculture et développement durable ». 
financé par l'Agence nationale de la recherche (ANR) qui porte sur six terrains d'investigation : Nouvelle-Calédonie, France métropolitaine, Brésil, Madagascar, Mali, et Mexique. Ces deux articles montrent ainsi, de manière très convergente, comment les dynamiques historiques, politiques et institutionnelles longues et les «bifurcations » (parfois brutales) expliquent la manière dont la problématique du DD, telle qu'exprimée dans les référentiels internationaux depuis le rapport Brundtland, émerge, est appropriée et concrètement mise en œuvre de manière différenciée au sein de territoires marqués par des caractéristiques rurales et agricoles spécifiques. Ils soulignent tous les deux la difficulté du changement institutionnel en faveur d'un développement plus durable : «L'évolution lente des mentalités des acteurs relève d'une logique de dépendance au sentier. Les décideurs politiques et les cadres administratifs continuent à concevoir les politiques comme relevant essentiellement d'interventions sectorielles et de fait la mise en place d'une politique intégrée de développement durable s'avère difficile tant les trajectoires de l'intervention publique en milieu rural sont liées aux représentations du monde que s'en font les acteurs du développement. » (Bosc et al. $)^{7}$

Ils insistent également sur la difficulté qu'il y a à mettre en place des politiques basées sur des principes généraux définis « de l'extérieur », dont la légitimité est d'autant plus interrogée qu' elle vient bousculer des trajectoires institutionnelles locales très prégnantes et des équilibres politiques instables ou fragiles. Comme le résument bien Jean-Michel Sourisseau et al. dans la conclusion de leur article : «Le cas calédonien vient finalement confirmer deux

7. Voir dans ce numéro l'article de Pierre-Marie Bosc, Marie-Hélène Dabat et Élodie Maître d'Hôtel, Quelles politiques de développement durable au Mali et à Madagascar ? phénomènes affectant l'intégration et la mise en ouvre du développement durable dans la ruralité et l'agriculture, observés dans d'autres pays [...] D'une part, le développement durable, du fait des ambiguïtés de sa définition et de sa portée idéologique, modifie de façon spécifique les débats politiques locaux. D'autre part, la superposition de l'injonction du développement durable à des dynamiques et idéologies antérieures - elles-mêmes encore en construction participe à l'explication des difficultés de sa mise en auvre. »

Dans les cas du Mali, de Madagascar, et celui de la Nouvelle-Calédonie (mais avec des difficultés supplémentaires dans ce dernier cas liées notamment aux liens privilégiés avec la France et aux logiques persistantes de rente minière), les deux articles montrent également comment les expressions variées des politiques de DD à l'œuvre dans ces territoires sont le produit spécifique de jeux d'acteurs politiques et de processus de négociation qui redessinent les rôles respectifs de l'État, du secteur privé et des communautés locales tout en faisant émerger de nouveaux acteurs collectifs (syndicats, associations professionnelles, communautés locales, etc.).

Tout en reflétant bien le nombre des aspects cruciaux évoqués ci-dessus, le cas du Parc du W étudié par Christophe Rouxel introduit deux dimensions supplémentaires qui compliquent davantage les choses : il s'agit, d'une part des problèmes (ethniques, culturels, politiques, etc.) posés par le caractère transfrontalier du parc, d'autre part de la question de la préservation de sa riche biodiversité suite à son classement comme « Réserve transfrontalière de biosphère » en 2002. Comme le souligne l'auteur sur le plan plus général des zones de savane en Afrique de l'Ouest : «De mosaïques écologiques, les zones de savanes ouest-africaines deviennent également des mosaïques d'enjeux territoriaux dans lesquels des transitions agraires et paysagères, différenciées selon les objectifs assignés, doivent être 
accélérées pour répondre favorablement à un développement durable local. »

La difficulté de la définition et de la mise en œuvre d'une stratégie équilibrée de développement de ces zones vient de ce que les trois piliers du développement durable (reformulés par l'auteur en termes « socio-territoriaux », « socio-économiques » et «agro-écologiques ») se télescopent plus brutalement encore que dans le cas général en raison des logiques très différenciées des acteurs en présence ${ }^{8}$, de la superposition de plusieurs régimes de droits de propriété foncière et de conflits d'usage de l'espace et des ressources particulièrement aigus au sein du territoire (notamment au niveau de sa «zone tampon »). Dans ce contexte, la crainte est forte que ce soit la logique d'une « gestion en mosaïque d'espaces-ressources » qui prévale et se pérennise, alors même que c'est probablement la combinaison de gestions intégrées des espaces-ressources de nature « agropastorale » et « agroforestière » qui semble la mieux à même d'assurer des compromis viables pour le développement durable du parc du W.

Deux des clés fondamentales d'une sortie par le haut que Christophe Rouxel identifie sont, d'une part, la participation active des populations locales (au niveau des villages ou des communes) à la préservation de la biodiversité, d'autre part, l'institutionnalisation d'instances de coordination efficace sur la base d'une gouvernance multilatérale : «À ces trois piliers [du développement durable, tels que reformulés par l'auteur] s'ajoute un enjeu transversal, la gouvernance, fondée sur la participation de tous les acteurs concernés à la

8. Pour un éclairage convergent dans le contexte des parcs naturels en France, voir l'analyse développée à partir du cas du Parc Naturel du Morvan par Parra C. (2010). Cf. Sustainability and Multilevel governance of territories classified as protected areas in France: the Morvan Regional Park, In Journal of Environmental Planning and Management, vol. 53, $\mathrm{n}^{\circ} 4$. définition d'un projet de secteur socioterritorial ou de zone tampon. »C'est ainsi que des «nattes de concertation, dont l'objectif premier est la résolution des conflits entre acteurs locaux permanents (agriculteurs) et ponctuels (éleveurs transhumants), et entre acteurs locaux et intervenants extérieurs (gestionnaires du W, services forestiers et concessionnaires de chasse), ont été initiées. Leurs membres pourront aussi être chargés de discuter de projets aptes à faciliter une transition agraire et paysagère de leurs territoires, en ayant soin de discuter des aspects favorables à un développement durable local. »

Cette insistance sur le caractère probablement local des concertations, négociations et solutions pouvant mener à un développement territorial viable est également présente dans les deux autres articles. Comme le soulignent Bosc et al. dans leur conclusion : "S'il est difficile voire impossible de construire au niveau national une politique cohérente de développement durable, le jeu des acteurs locaux, en prise directe avec les problèmes sociaux et environnementaux concrets, peut être un moyen de prendre en compte les différentes dimensions de la durabilité de manière plus intégrée. » Pour leur part, Jean-Michel Sourisseau et al. insistent également sur le caractère différencié de l'appropriation et de la mise en œuvre des orientations du développement durable par les collectivités locales calédoniennes.

Au total, les articles réunis dans ce dossier reflètent bien le rôle crucial, d'une part, des spécificités culturelles, institutionnelles, socio-économiques et politiques héritées des trajectoires historiques différenciées de territoires aux ressources et conditions naturelles elles-mêmes particulières, d'autre part, des jeux et compromis d'acteurs qui se nouent de manière plus ou moins efficace et viable à certaines échelles spatiales et à certains moments particuliers. Parallèlement, mais sans que cela ne soit explicitement développé dans les différents articles, 


\section{INTRODUCTION}

l'évolution des conceptions du développement durable d'un territoire et des actions engagées pour sa mise en œuvre concrète relèvent, probablement de manière tout aussi fondamentale, de processus cognitifs et de mécanismes d'apprentissage individuels et collectifs essentiellement localisés ${ }^{9}$. Ce sont vraisemblablement ces processus qui permettent de faire émerger des modes prati- cables de gouvernance et de résolution des conflits (de visions, d'objectifs, d'usages, de rationalités) afin de privilégier des orientations de développement territorial dans un sens marqué de durabilité et d'équilibre viable (i.e., acceptable pour les populations et les différentes parties prenantes) entre ses dimensions économique, sociale et environnementale.

9. Sur ce point, lire l'analyse éclairante de Singh R., Pretty J., Pilgrim S. (2010) dans le contexte des communautés rurales en Inde, et tout particulièrement du rôle central qu'y jouent les femmes dans la transmission de connaissances et de pratiques (souvent ancestrales) favorables à un développement territorial durable. $C f$. Traditional knowledge and biocultural diversity learning from tribal communities for sustainable development in northeast India, Journal of Environmental Planning and Management, vol. 53, $\mathrm{n}^{\circ} 4$. 